Trauma Berufskrankh 2005 · 7[Suppl 1]:S225-S230 DOI 10.1007/s10039-004-0864-y

Online publiziert: 22. April 2004

(c) Springer Medizin Verlag 2004

\title{
J. Zaage
}

Klinik für Allgemein- und Viszeralchirurgie der Berufsgenossenschaftlichen Kliniken

Bergmannstrost, Halle/Saale

\section{Das abdominelle Kompartmentsyndrom}

Diese pathophysiologischen Veränderungen sind in vielen Untersuchungen bestätigt worden, allerdings mit einer Ausnahme: Bei Vorliegen einer Hypervolämie fand sich im Gegensatz zu Hypo- oder Normovolämie ein Anstieg des Herzzeitvolumens. Erklärt wird dieses Phänomen dadurch, dass bei Hypervolämie der Druck in der unteren Hohlvene höher ist und dadurch der venöse Rückstrom weniger beeinträchtigt wird. Letztlich kommt es jedoch im Sinne eines Circulus vitiosus zur weiteren abdominellen Druckerhöhung bei gleichzeitigem venösen Stau.

Lunge. Die pulmonalen Veränderungen werden hauptsächlich durch eine mechanische Behinderung der Atmung ausgelöst. Durch den Zwerchfellhochstand kommt

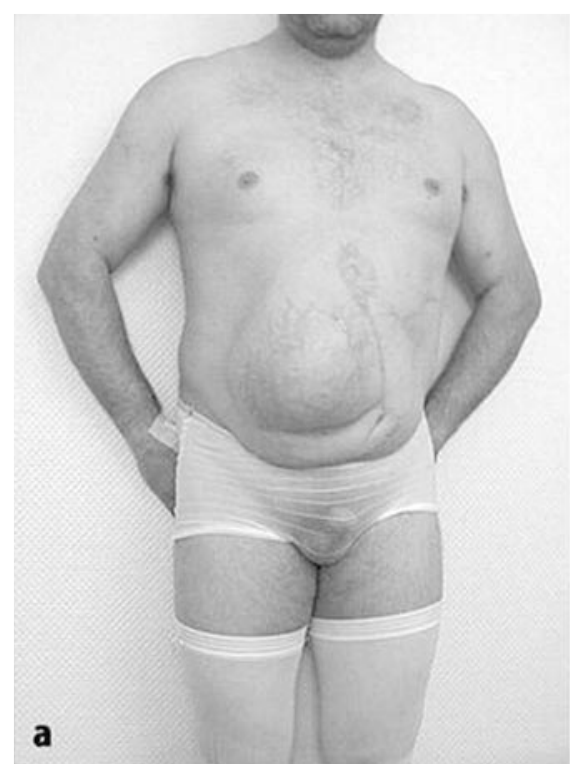

Abb. $1 \Delta$ Monströse Bauchwandhernie es zur Kompression der Lungen mit Anstieg des pleuralen Druckes und einer Abnahme der Compliance. In der Folge entwickeln sich eine Abnahme des SauerstoffPartialdruckes und eine gleichzeitige $\mathrm{Zu}$ nahme des $\mathrm{CO}_{2}$-Partialdruckes.

Niere. Das Ausmaß der Nierenfunktionsbeeinträchtigung hängt entscheidend vom systemischen Blutdruck ab. Die Oligurie entsteht bei gleichbleibender Nierenperfusion durch die Abnahme des Filtrationsgradienten über dem Glomerulum. Die Abnahme der Urinproduktion lässt sich nicht durch eine direkte Kompression der Ureteren erklären, weil eine Ureterenschienung nicht zu einer Zunahme der Urinausscheidung führt. Diskutiert werden aber eine direkte Kompression der Nieren und

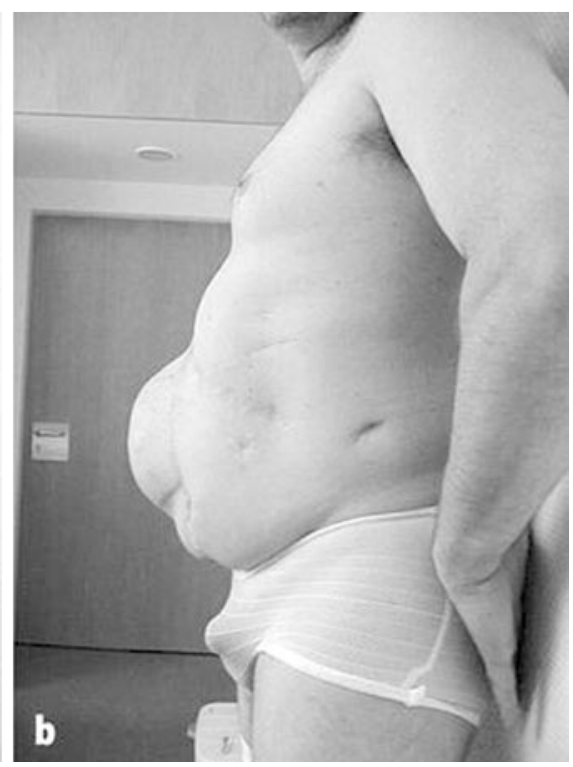
und Kompression des Herzens und der großen intrathorakalen Gefäße. 
Tabelle 1

Pathophysiologische Auswirkungen der intraabdominellen Druckerhöhung und klinische Folgen

\begin{tabular}{|lll}
\hline $\begin{array}{l}\text { Organ } \\
\text { Herz/Kreislauf }\end{array}$ & Pathophysiologischer Effekt & Klinisches Symptom \\
& $\begin{array}{l}\text { Venöser Rückstrom sinkt } \\
\text { Herzzeitvolumen nimmt ab } \\
\text { Peripherer Widerstand steigt }\end{array}$ & \\
\hline Lunge & $\mathrm{pO}_{2}$ nimmt ab & \\
& $\mathrm{pCO}_{2}$ nimmt zu & \\
& Compliance sinkt & Respiratorische Insuffizienz \\
& Pleuraler Druck steigt & Hypoxie \\
& Beatmungsdruck steigt & Hyperkapnie \\
& Glomeruläre Filtration sinkt & \\
\hline Niere & Durchblutung sinkt & Oligurie \\
& Perfusionsstörung & Anurie \\
\hline Leber/Darm & Translokation & Leberversagen \\
& Azidose & Intestinale Ischämie \\
& SIRS & Paralyse \\
& Darmnekrose & Distension \\
& Venöser Abstrom sinkt & \\
& Perfusion sinkt & Zerebrale Ischämie \\
& Hirndruck steigt & \\
\hline ZNS & & \\
& &
\end{tabular}

Tabelle 2

\section{Häufigste Ursachen des abdominellen Kompartmentsyndroms}

\begin{tabular}{ll} 
Kategorie & Ursache \\
Akut, posttraumatisch & Stumpfes Bauchtrauma \\
& Penetrierendes Bauchtrauma \\
& Intraperitoneale Blutung \\
& Retroperitoneale Blutung \\
& Darmwandödem durch Endothel-Leak nach Ischämie/Reperfusion \\
& und aggressiver Volumentherapie \\
& Verminderte Bauchwandcompliance nach Verbrennung \\
\hline Akut, postoperativ & Nachblutung nach Laparotomie \\
& Abdominelles Packing (Kavakompression) \\
& Koagulopathie \\
& Darmwandödem nach Ischämie/Reperfusion \\
& Akute Pankreatitis \\
& Peritonitis \\
Intraabdominelle Abszessbildung \\
Ileus \\
Pneumoperitoneum \\
Bauchdeckenverschluss unter Spannung \\
Hernienverschluss bei großen Hernien unter Spannung \\
\hline Blutung \\
Koagulopathie \\
Aortenaneurysmaruptur \\
Aszites \\
Tumor \\
Schwangerschaft \\
\hline Sonstige (spontan) & \\
\hline Chronisch & \\
& \\
& \\
&
\end{tabular}

Tabelle 3

Definition des intraabdominellen

Kompartmentsyndroms (AKS)

Intraabdominelle Druckerhöhung (IAD)

Unterschiedlichste Pathogenese

Störung der Mikrozirkulation

Störung der Makrozirkulation

Organversagen

Multiorganversagen

Tod

$A K S=I A D \uparrow+$ Organversagen

hormonelle Faktoren. Welche Rolle jedoch ADH, Renin und Angiotensin wirklich spielen, ist noch nicht geklärt.

Leber/Darm. Sowohl in Tierexperimenten als auch bei laparoskopischen Eingriffen konnte eine intestinale Ischämie durch den intraabdominellen Überdruck nachgewiesen werden. Es entsteht nicht nur eine Flussminderung in der peripheren mesenterialen Strombahn, sondern auch im Truncus coeliacus und in der A. mesenterica superior.

Somit führt die Druckerhöhung im Splanchnikusbereich zu einer Verschlechterung der Zirkulation in Leber und Darm. Letztlich kommt es durch die gestörte Perfusion zur lokalen Azidose, Schleimhautschädigung und damit ggf. zu Translokationsphänomenen. Diese Auswirkungen auf die Leber- und Darmfunktion verstärken sich noch, wenn als Ursache schon eine Perfusions- oder Barrierestörung vorliegt - wie eben z. B. durch Schock beim Polytrauma mit Abdominalbeteiligung, bei Vorliegen einer Peritonitis oder durch intensivmedizinische Behandlung ganz allgemein. Beispielhaft seien genannt Volumen- und Katecholamingabe. Dann kommt es zu einem zusätzlichen Darmwandödem, zu Darmparalyse, Bakterienüberwucherung und progressivem septischen Organversagen.

ZNS. Ein erhöhter intraabdomineller Druck hat auch Einfluss auf den intrakraniellen Druck. Nachgewiesen ist sowohl experimentell als auch klinisch, dass die zerebrale Perfusion bei erhöhtem intraabdominellem Druck abnimmt, was zumindest teilweise durch einen verminderten 


\section{Zusammenfassung $\cdot$ Abstract}

venösen Hirnabfluss erklärt werden kann. Letztlich sind aber die Zusammenhänge noch nicht vollständig aufgeklärt.

ZVD-Messung. Wenn wir von pathophysiologischen Auswirkungen sprechen, so darf nicht vergessen werden, dass der intraabdominelle Hochdruck auch die Verlässlichkeit der Messung sowohl des zentralvenösen Druckes (ZVD) als auch des pulmonalen, kapillären Wedge-Druckes beeinflusst. Durch den teilweise sogar stark erhöhten pleuralen Druck können diese Werte sehr hoch sein, obwohl das intravasale Volumen eben niedrig ist!

\section{Ursachen des abdominellen Kompartmentsyndroms}

Was sind nun die Ursachen, die zu einem abdominellen Kompartmentsyndrom führen können? Im Wesentlichen lassen sie sich in posttraumatische, postoperative und sonstige Ursachen einteilen. Im Vordergrund stehen dabei das Polytrauma, die große Bauchoperation und die Aortenersatzoperation (• Tabelle 2 ). Hier soll nur auf einige Besonderheiten eingegangen werden

Schwerstbrandverletzte. Schwerstbrandverletzte weisen 2 Besonderheiten auf: eine mechanische - und zwar die verminderte Compliance der Bauchwand durch Narbenbildung - und eine pathophysiologische:Diese Patienten brauchen über Monate hin eine teilweise aggressive Intensivtherapie. Damit sind aber die Folgen eines interkurrent auftretenden abdominellen Kompartmentsyndroms besonders dramatisch.

Packing. Beim Packing des Abdomens allgemein, insbesondere aber der Leber handelt es sich um eine sehr segensreiche Blutstillungsmethode - aber nur bei richtiger Technik. Die 2 häufigsten Fehler dabei sind:

- eine Tamponade direkt in die Läsion hinein und

- eine Tamponade von vorn und unter bzw. über die Leber.

Eine suffiziente Lebertamponade ist nur möglich, wenn das Ligamentum triangu-

Trauma Berufskrankh $2005 \cdot 7[$ Suppl 1]:S225-S230

DOI 10.1007/s10039-004-0864-y

c) Springer Medizin Verlag 2004

J. Zaage

\section{Das abdominelle Kompartmentsyndrom}

\section{Zusammenfassung}

Das abdominelle Kompartmentsyndrom ist ein klar definiertes Krankheitsbild, das aber leider noch zu wenig Beachtung findet. Obwohl vor fast 100 Jahren schon beschrieben, wurde es als Krankheitsentität erst in den letzten Jahren akzeptiert. Die möglichen Ursachen sind vielfältig und beruhen auf einer abdominellen Volumenvermehrung. Diese führt zu Störungen mehrerer Organsysteme wie Herz/Kreislauf, Lunge, Niere, Leber, Darm und ZNS bis hin zum Multiorganversagen. Nur die möglichst frühzeiti-

\section{Abdominal compartment syndrome}

\section{Abstract}

Abdominal compartment syndrome has a clearly defined clinical appearance which, in my opinion, does not receive enough attention. Although described nearly 100 years ago, it has only gained acceptance in the last few years. The probable causes are various and are based on an abdominal volume increase that leads to disorders in multiple organs and systems: cardiac, circulatory, pulmonary, renal, hepatic, intestinal and central nervous, up to multiple organ failure. Only detection followed by surgical ab- ge Erkennung und Behandlung durch eine chirurgische Dekompression kann den ansonsten letalen Verlauf verhindern. Bei Risikosituationen sollte deshalb im Zweifelsfall das Abdomen nicht primär verschlossen, sondern offen weiterbehandelt werden.

\section{Schlüsselwörter}

Abdominelles Kompartmentsyndrom . Multiorganversagen - Chirurgische Dekompression · Abdominelle Volumenvermehrung dominal decompression as early as possible can prevent an otherwise lethal outcome. In risk situations, if in doubt, the abdomen should not be closed primarily, it should rather be left open until closure can be safely performed.

\section{Keywords}

Abdominal compartment syndrome . Multiple organ failure · Surgical abdominal decompression - Abdominal volume increase 


\section{Intensivmedizin des Polytraumas}

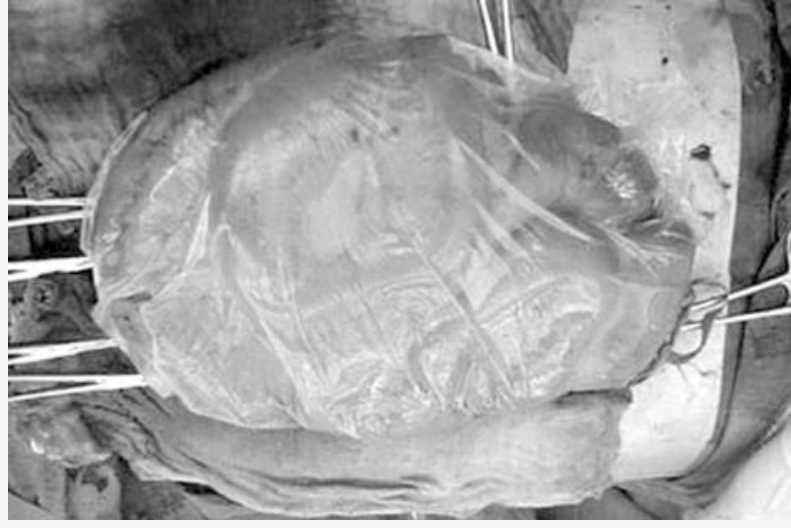

Abb. $2 \Delta$ Provisorischer Bauchdeckenverschluss nach therapeutischer Dekompression des Abdomens

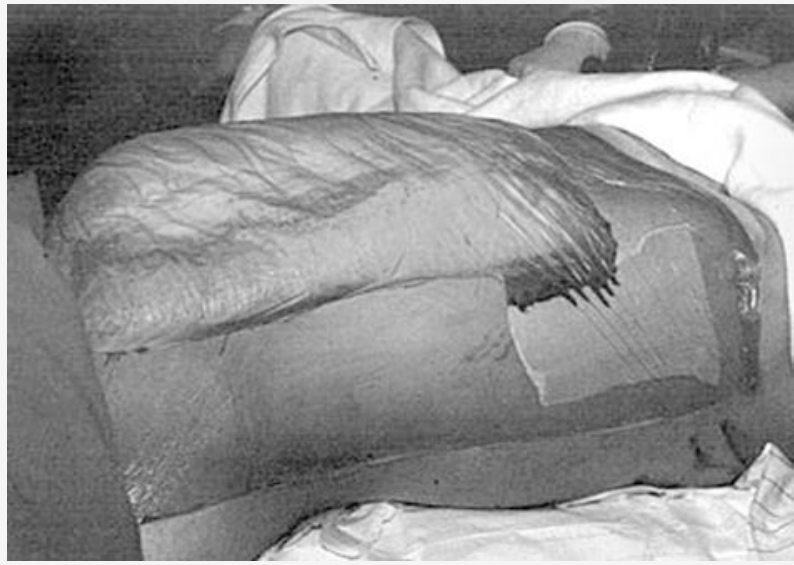

Abb. 4 A Provisorischer Bauchdeckenverschluss (nach 24 h)

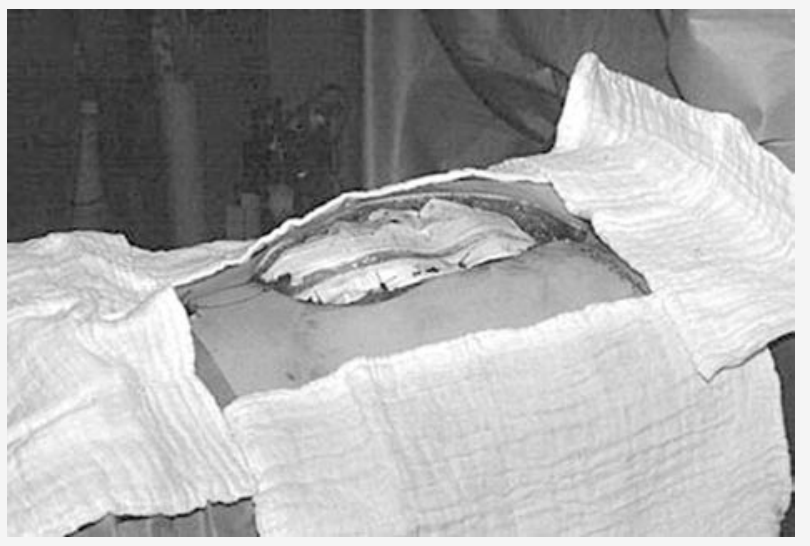

Abb. $6<$ Provisorischer Bauchdeckenverschluss (nach 48 h, Verfahrenswechsel) lare - und zwar rechts wie links - ausreichend inzidiert wird, um neben der V. cava inferior auch von hinten ein Widerlager zu schaffen.

\section{Bauchdeckenverschluss unter Spannung.}

Dies kann besonders nach großen, länger dauernden Bauch- oder Gefäßoperationen zum Problem werden. Meist ist dabei schon während der Operation ein zuneh-

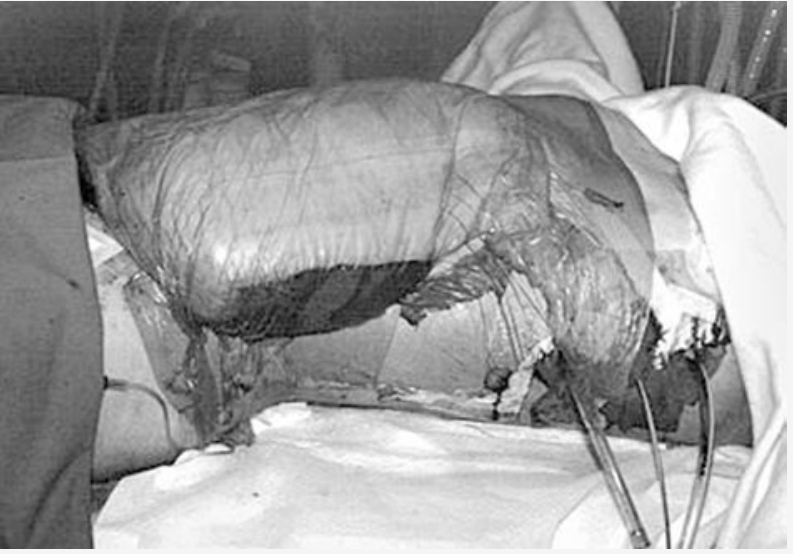

Abb. 3 Provisorischer Bauchdeckenverschluss (nach 12 h)

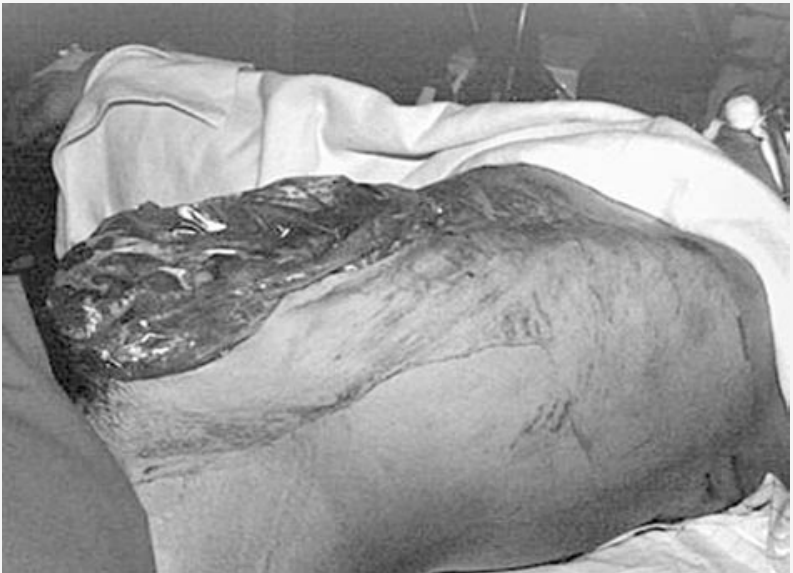

Abb. 5 Provisorischer Bauchdeckenverschluss (nach 48 h) mendes Darmwandödem zu registrieren, das dann auch die Sichtverhältnisse beeinträchtigt. In diesen Fällen ist es besser, das Abdomen primär offen zu lassen. Meist lässt sich bei adäquater Intensivtherapie der Bauchdeckenverschluss schon nach 12 Tagen spannungsfrei durchführen.

Hernienverschluss. Diese Ursache wurde aus der Literatur übernommen. Sie sollte in diesem Zusammenhang jedoch gar nicht erwähnt werden müssen. Denn solche monströsen Bauchwandhernien sollte nur der operieren, der ausreichend Erfahrung hat und alle zur Verfügung stehenden Operationsmethoden beherrscht ( $\bullet$ Abb. 1).

Abgebildet ist ein 38jähriger Patient mit einer Rezidivnarbenhernie nach 8 Voroperationen wegen eines Unfalles von 1987 mit Bauchtrauma. Wir haben die Versorgung 
mit einer Kombination verschiedener Verfahren vorgenommen: der Komponentenseparation nach Ramirez, einer Türflügelplastik und einer Vicrylnetzimplantation.

Pathogenese. Die häufigsten und frühen Beschreibungen des abdominellen Kompartmentsyndroms hatten eine Blutung zum Gegenstand. Das häufigere Auftreten durch Überwindung der Schockphase bei Traumapatienten, auf Intensivstationen mit aggressiver Volumentherapie und bei inadäquater Tamponade haben zusammen mit der Kenntnis des Ischämie-Reperfusionsschadens und der Pathophysiologie des Pneumoperitoneums zur Akzeptanz des abdominellen Kompartmentsyndroms als Krankheitsentität geführt. Wir verstehen unter einem abdominellen Kompartmentsyndrom eine intraabdominelle Druckerhöhung unterschiedlichster Pathogenese, die letztlich jedoch - wie auch bei anderen Kompartmentsyndromen - zur Beeinträchtigung der Mikrozirkulation führt. Die Kompromittierung der abdominellen wie auch der retroperitonealen Organe in Verbindung mit den makrozirkulatorischen Folgen führen zum Multiorganversagen und schließlich zum Tode.

Man könnte somit auch sagen: Ein abdominelles Kompartmentsyndrom ist ein Organversagen infolge einer intraabdominellen Druckerhöhung (• Tabelle 3).

\section{Intraabdominelle Druckmessung}

Maßeinheiten. Der normale intraabdominelle Druck ist gleich oder niedriger als der atmosphärische Druck, beim beatmeten Patienten entspricht er dem endexspiratorischen Druck. In allen Lehrbüchern steht, dass eine Veress-Nadel zur Anlage eines Pneumoperitoneums dann richtig in der freien Bauchhöhle platziert ist, wenn der Druck unter $10 \mathrm{mmHg}$ liegt. Wer laparoskopiert, weiß, dass es bei richtiger $\mathrm{Na}$ dellage sogar gelingt, einen Unterdruck $\mathrm{zu}$ erzeugen. So ist ein Druck um die o$10 \mathrm{mmHg}$ als normal zu bezeichnen. Ab $10 \mathrm{mmHg}$ hat Meldrum [2] 4 Schweregrade definiert (• Tabelle 4 ). In vielen anderen Arbeiten wird die intraabdominelle Hypertension definiert als ein andauernder Druck über $20-25 \mathrm{~cm} \mathrm{H}_{2} \mathrm{O}$.

Nun haben wir also eine zweite Druckmessangabe, nämlich außer mmHg noch

\section{Tabelle 4}

Schweregrade des abdominellen Kompartmentsyndroms (Nach Meldrum [2])

\begin{tabular}{|lll}
\hline Schweregrad & Druck $(\mathbf{m m H g})$ & Therapie \\
1 & $10-15$ & Normovolämie, Diurese \\
\hline 2 & $16-25$ & Aggressive Volumentherapie \\
\hline 3 & $26-35$ & Dekompression \\
\hline 4 & $>36$ & Dekompression und Reexploration \\
\hline & & Tabelle5
\end{tabular}

$\mathrm{cm} \mathrm{H}_{2} \mathrm{O}$. Bei der Vorbereitung auf die heutige Veranstaltung ist mir aufgefallen, dass selbst innerhalb einer Arbeit manche Autoren beide Messangaben losgelöst voneinander gebrauchen.

Deshalb sind hier die Umrechnungen zusammengestellt worden, wobei auf 2 Stellen nach dem Komma gerundet wurde (- Tabelle 5). Der jetzt interessierende Bereich von $20-25 \mathrm{~cm} \mathrm{H}_{2} \mathrm{O}$ entspricht in etwa 15-20 mmHg und wäre nach Meldrum bereits Schweregrad 2 zuzuordnen.

Vorgehen. Wie nun kann man aber überhaupt den intraabdominellen Druck messen? Die direkte Druckmessung beruht auf dem Veress-Nadel-Prinzip. Wir halten sie bei den ohnehin meist schwerkranken $\mathrm{Pa}$ tienten für zu invasiv und haben damit deshalb bisher keine eigenen Erfahrungen.

Die indirekte Messung erfolgt über den Harnblasendruck. Zum Messzeitpunkt wird die Harnblase über den Harnblasenkatheter entleert und dann mit $100 \mathrm{ml}$ einer sterilen isotonen Lösung aufgefüllt. Anschließend kann die Höhe der Wassersäule in $\mathrm{cm}$ über dem Schambein abgelesen werden. Dabei kommt es nicht immer nur auf den absoluten Messwert an, vielmehr ist der Druckverlauf manchmal noch wichtiger, v. a. in Korrelation zum klinischen Bild.

Die Klinik ist geprägt durch ein geblähtes, häufig schmerzhaft gespanntes Abdomen. Charakteristisch dazu ist eine zunehmend inadäquate Ventilation sowie eine Störung der Nierenfunktion. Allerdings wird die klinische Symptomatik durch die primäre Schädigung mitbedingt oder sogar bestimmt, weshalb zunächst einmal das „Darandenken“ in entsprechenden Risikosituationen entscheidend ist.

\section{Therapie}

Therapie der Wahl ist die chirurgische Dekompression, die einer therapeutischen La-
Druckvergleichswerte

\begin{tabular}{rlc}
\hline $1 \mathrm{mmHg}$ & $=$ & $1,359 \mathrm{~cm} \mathrm{H}_{2} \mathrm{O}$ \\
\hline $10 \mathrm{mmHg}$ & $=$ & $13,59 \mathrm{~cm} \mathrm{H}_{2} \mathrm{O}$ \\
\hline $15 \mathrm{mmHg}$ & $=$ & $20,39 \mathrm{~cm} \mathrm{H}_{2} \mathrm{O}$ \\
\hline $20 \mathrm{mmHg}$ & $=$ & $27,18 \mathrm{~cm} \mathrm{H}_{2} \mathrm{O}$ \\
\hline $25 \mathrm{mmHg}$ & $=$ & $33,98 \mathrm{~cm} \mathrm{H}_{2} \mathrm{O}$ \\
\hline $1 \mathrm{~cm} \mathrm{H} \mathrm{O}$ & $=$ & $0,74 \mathrm{mmHg}^{-}$ \\
\hline $10 \mathrm{~cm} \mathrm{H}_{2} \mathrm{O}$ & $=$ & $7,35 \mathrm{mmHg}$ \\
\hline $15 \mathrm{~cm} \mathrm{H}_{2} \mathrm{O}$ & $=$ & $11,03 \mathrm{mmHg}^{-}$ \\
\hline $20 \mathrm{~cm} \mathrm{H}_{2} \mathrm{O}$ & $=$ & $14,70 \mathrm{mmHg}$ \\
\hline $25 \mathrm{~cm} \mathrm{H}_{2} \mathrm{O}$ & $=$ & $18,38 \mathrm{mmHg}$ \\
\hline
\end{tabular}

parotomie entspricht. Die Indikation dazu wird wesentlich vom klinischen Bild des Patienten beeinflusst. Bei zwar wenig dramatischen Befunden, aber entsprechender Risikokonstellation mit Organdysfunktion sollte zumindest bei einem Druck über $25 \mathrm{mmHg}$ die Laparotomie erfolgen. Großzügiger noch sehen wir die Indikation bei gleichzeitigem Schädel-Hirn-Trauma, bei Schwerstbrandverletzten oder bei Nachblutungen mit der Möglichkeit einer gezielten Blutstillung oder Tamponade. Ebenfalls großzügig sind wir bei der Annahme einer technisch mangelhaften Tamponade mit Kavakompression.

In seltenen Fällen, wie z. B. bei Aszites, kann man die Bauchhöhle drainieren und verschließen. Meist ist jedoch die weitere offene Behandlung des Abdomens erforderlich. Für einen provisorischen Verschluss der Bauchdecke kommen verschiedene Möglichkeiten in Betracht, wie z. B. Reißverschluss, Vicrylnetz oder - wie von uns bevorzugt - Plastikfolien ( $\bullet$ Abb. 2, 3, 4, 5, 6). Gegebenenfalls kann man den Bauch nach Reexploration definitiv verschließen. In Extremfällen muss jedoch das Abdomen notfalls auch über längere Zeit offen weiterbehandelt werden. 
Tabelle 6

Paraklinische Parameter beim abdominellen Kompartmentsyndrom vor und nach chirurgischer Dekompression

\begin{tabular}{|c|c|c|c|c|c|c|c|c|c|c|}
\hline \multirow[t]{2}{*}{ Patient } & \multicolumn{2}{|c|}{$\begin{array}{l}\text { Harnblasendruck } \\
\left(\mathrm{cm} \mathrm{H}_{2} \mathrm{O}\right)\end{array}$} & \multicolumn{2}{|c|}{$\begin{array}{l}\text { Urinausscheidung } \\
(\mathrm{ml} / \mathrm{h})\end{array}$} & \multicolumn{2}{|c|}{$\begin{array}{l}\text { Kreatininwert } \\
(\mathrm{mmol} / \mathrm{l})\end{array}$} & \multicolumn{2}{|c|}{$\begin{array}{l}\mathrm{paO}_{2} \\
(\mathrm{~Pa})\end{array}$} & \multicolumn{2}{|c|}{$\begin{array}{l}\mathrm{paCO}_{2} \\
(\mathrm{~Pa})\end{array}$} \\
\hline & vor $^{\mathrm{a}}$ & nach $^{b}$ & vor $^{\mathrm{a}}$ & nach $^{\mathbf{b}}$ & vor $^{\mathrm{a}}$ & nach $^{\mathbf{b}}$ & vor $^{\mathrm{a}}$ & nach $^{\mathbf{b}}$ & vora $^{\mathrm{a}}$ & nach $^{\mathbf{b}}$ \\
\hline 1 & 48 & 29 & 20 & 80 & 79 & 53 & 7,8 & 12,4 & 7,1 & 6,3 \\
\hline 2 & 33 & 22 & 35 & 50 & 48 & 48 & 8,1 & 10,4 & 5,7 & 4,4 \\
\hline 3 & 36 & 25 & 10 & 25 & 189 & 209 & 8,8 & 10,4 & 6,4 & 6,1 \\
\hline 4 & 43 & 29 & 20 & 80 & 100 & 58 & 12,4 & 9,0 & 5,7 & 3,9 \\
\hline 5 & 33 & 17 & 15 & 110 & 58 & 58 & 7,4 & 10,0 & 5,4 & 5,4 \\
\hline 6 & 32 & 24 & 20 & 150 & 199 & 235 & 9,6 & 8,4 & 5,4 & 5,2 \\
\hline 7 & 42 & 17 & 10 & 45 & 133 & 171 & 6,3 & 9,3 & 4,7 & 4,0 \\
\hline \multicolumn{11}{|c|}{${ }^{a}$ vor Dekompression } \\
\hline
\end{tabular}

Gleichzeitig zur operativen Dekompression sollten verschiedene Maßnahmen zur Reduktion eines Darmwandödems und einer Darmparalyse ergriffen werden. Dazu zählen:

- eine angepasste, situationsgerechte Volumen- und Katecholamintherapie.

- eine forcierte Diurese oder evtl. eine

- Hämofiltration, die Verabreichung von

- Prokinetika, um die Darmtätigkeit anzuregen,

- das Legen einer Magensonde und

- die Vermeidung von Medikamenten mit negativen Nebeneffekten, wie etwa eine unkritisch hohe Analgosedierung oder Relaxation.

Die effektivste Maßnahme aber bleibt sicherlich die Prävention!

\section{Prävention}

An erster Stelle steht hier der spannungsfreie Bauchdeckenverschluss. Ein Faszienverschluss - noch dazu unter Relaxierung - sollte keinesfalls erzwungen werden.

In Risikosituationen wie beim rupturierten Aortenaneurysma, beim schweren Abdominaltrauma, bei Schwerstbrandverletzten oder bei besonderen Peritonitisformen sollte erwogen werden, auf einen primären Bauchdeckenverschluss ganz zu verzichten.

\section{Patientendaten}

In $\bullet$ Tabelle 6 sind die Originaldaten von 7 Patienten mit einem abdominellen Kompartmentsyndrom dargestellt. Beachtung finden sollten v. a. die Messwerte jeweils vor und 30-6o min nach Dekompression.

Hingewiesen sei nur auf 3 Dinge:

1. Alle Patienten hatten einen eindeutig erhöhten intraabdominellen Druck.

2. Nach Dekompression verbesserte sich die Situation schlagartig in Bezug auf den intraabdominellen Druck, die Urinausscheidung und die Gasaustauschwerte.

3. Die Kreatininwerte können teilweise sogar noch weiter ansteigen und fallen erst nach einigen Tagen ab.

\section{Fazit für die Praxis}

Das abdominelle Kompartmentsyndrom beruht auf einer Zunahme des Bauchvolumens. Zur Messung des erhöhten intraabdominellen Drucks empfiehlt sich die indirekte Messung über den Harnblasendruck. Dabei wird die Höhe der Wassersäule in cm über dem Schambein abgelesen. Sowohl der absolute Messwert als auch der Druckverlauf sollten beobachtet werden. Wird das Syndrom nicht frühzeitig erkannt, ist sein Verlauf letal. Die chirurgische Dekompression ist die Therapie der Wahl. Vorzubeugen ist der Erkrankung durch einen spannungsfreien Bauchdeckenverschluss. Ein Faszienverschluss sollte keinesfalls erzwungen werden. Bei besonders risikobehafteten Krankheitsbildern, z. B. dem rupturierten Aortenaneurysma, sollte ggf. auf einen primären Bauchdeckenverschluss ganz verzichtet werden.

\section{Korrespondierender Autor}

\section{Dr.J.Zaage}

Klinik für Allgemein- und Viszeralchirurgie der Berufsgenossenschaftlichen Kliniken Bergmannstrost, Merseburger Straße 165, 06112 Halle/Saale

Interessenkonflikt: Der korrespondierende Autor versichert, dass keine Verbindungen mit einer Firma, deren Produkt in dem Artikel genannt ist, oder einer Firma, die ein Konkurrenzprodukt vertreibt, bestehen.

\section{Literatur}

1. Emerson $\mathrm{H}$ (1911) Intra-abdominal pressures. Arch Intern Med 7: 754-784

2. Meldrum DR, Moore FA, Moore FF et al. (1997) Prospective characterization and selective management of the abdominal compartment syndrome. Am J Surg 174: 667-673

3. Thorington JM, Schmidt CF (1923) A study of urinary output and bloodpressure changes resulting in experimental ascites. Am J Med Sci 165: 800-805 